\title{
The Report of School Violence Platform
}

\author{
Junke Zhuang ${ }^{1, *,+}$, Zhipian $\mathrm{Lu}^{2,+}$
}

\author{
${ }^{1}$ Collège du Léman, Versoix, 1290, Switzerland \\ ${ }^{2}$ University Colledge London, London, UK \\ *Correspondence author:Junke_zhuang@cdl.ch \\ + They are all share first author.
}

\begin{abstract}
School bullying is a growing concern in Chinese society. However, measures to protect and support school bullying victims are inadequate. Therefore, a market opportunity of the application, that focus on victim's online interaction and provision of psychological counselling services was recognised by researchers. This project aimed to recognise and test the desirability risk, viability risk, and feasibility risk of the purposed friend-making platform for school bullying victims using interviews and survey. The research found that the victims demonstrated their desires to have the application and the likelihood for schools to fund the project was low. Moreover, viability risk of the revenue needed to be tested further as the expected monthly cost of most respondents is low. Further research is needed to explore other viability, desirability and feasibility risk of the social venture.
\end{abstract}

Keywords: School bullying, victim, application

\section{INTRODUCTION}

The negative impacts on victims' physical and mental health caused by school bullying have gradually drawn wide attention from society. Regarding this, the national level also has repeatedly voiced clear governance. In 2017, the General Office of the State Council issued a rule on strengthening the construction of a safety defense system in schools. In 2018, the Chinese education ministry defined governance solutions, while some local governments issued the bullying prevention or treatment plan, aiming to solve the problem of campus bullying and to optimize the environment of school safety.

Despite the national attention of the government, school bullying is still a serious issue among children and teenagers. School bullying has negative short-term and long-term impacts on victims' wellbeing, ranging from physical health to mental health. In terms of physical health, victims of school bullying will experience physical pain and psychological stress and might develop a series of somatic complaints [1]. Moreover, victims of school bullying can experience internalising issues such as depression, anxiety, and low self-esteem [2]. However, so far, no apps serve for school bullying. Therefore, we developed an application which targets at victims of school bullying and mental harm brought by it.

\subsection{The APP has three main functions:}

- to provide professional psychological counselling on campus bully, especially for the bullied;

- to provide a platform for users to interact with each other;

- to provide a platform for users to obtain voluntary help from those who experienced campus bullying but have recovered from it.

\section{LITERATURE REVIEW}

Limited empirical studies on campus violence can be found in mainland China. In Chan and Wong (2015)'s study, they explored the characteristics of bullying in China and used collectivist culture value to explain the common type of peer victimisation- social exclusion [3]. Socialisation contributes to the valuation of the interests of groups over that of individuals. Therefore, school bullying is used as collective conduct to maintain group conformity in China [3]. Research also found that the underreporting cases of school bullying in Taiwan due to interpersonal harmony values stressed by culture [4]. The values encourage students to increase self-control and tolerance which lead to under-reporting cases by students 
and staffs. Chan and Wong (2015) find that the prevalence rate of self-reported peer victimisation ranged from $2 \%$ to $66 \%$ in Mainland China [3]. A more recent paper found that the incidences of self-reported bullying victimisation are $26 \%$, in which "make fun of" and "spread rumour" are the top two bullying behaviours [5].

Studies on bullying prevention programmes in mainland China are limited and the mental health problem of victims are ignored. Zhang (2016) conducted a five-week school bullying intervention programme and found a remarkable reduction in both the incidence and the severity of victimisation with the intervention programme [6]. Further, the government published a guiding brief on school bullying prevention and intervention for schools and parents, but the implication and effectiveness of the guidebook are questionable [7]. $\mathrm{Xu}$ and colleagues (2016) argued that school bullying intervention organisations can hardly be found in China [8]. The ability to protect victims from bullying is limited due to the vague responsibilities of school administrators.

In terms of government's intervention or prevention, the government published some school-bullying-related laws, such as in the Constitution and Laws on the Protection of Minors. However, there is no law which only focuses on school bullying in China [9]. In terms of school measures for school bullying, some schools follow the guidance of the Education Ministry and provide psychology lessons and group work to prevent school bullying [10]. However, the effectiveness of these measures remains unclear.

In terms of the private sector, some applications such as "One psychology" or "Simply Psychology" provide online psychological counselling services for people. And hospitals and private clinics also provide in-person counselling or therapy for people with mental health problems. But there is no private sector that only focuses on victims of school bullying.

This research identified a gap of applications and measures in supporting school bullying victims, which provides (1) victim-only friend-making services; (2) psychological counselling services. Emotional support from friends is protective to reduce victims' internalising symptoms [12]. Moreover, psychological counselling services help diagnose symptoms and victims' recovery. Therefore, an application that involves emotional supports from both users and experts can be helpful for bullying victims' psychological wellbeing.

\section{METHOD}

To understand the risks of the platform with a focus on school bullying and to make more adequate preparations, we made assumptions from three aspects, desirability, feasibility, and viability.

\subsection{Desirability}

Hypothesis 1: People with school bullying experience want to have a platform to express their emotion.

\subsection{Viability}

Hypothesis 2: Schools are willing to fund and pay for the services related to school bullying.

Hypothesis 3: Victim of school bullying are willing to pay 30 yuan per month for the services.

\subsection{Feasibility}

Hypothesis 4: The platform can gain profit from each consultation, by $20 \%$ of the price of the counselling services.

Further, this study also explored interviewees' preferred design of the platform, including features to pay, recovered victims' willingness and motivations to help, privacy consideration and interactive modes.

This study was conducted using a survey to test the desirability (Hypothesis 1) and viability risks (Hypothesis 3) to understand potential consumers' attitudes towards the platform and services. Moreover, Hypothesis 2 and 4 were tested by interviewing four school teachers and a psychologist, respectively.

\section{RESULTS}

Results of the survey showed that one-third (30) of 105 respondents have been bullied (including those who have been bullied and those who have recovered from bully experience).

Among 30 victims of school bullying, $76.7 \%$ of them reported that they have been bullied via language bullying, while $66.7 \%$ experienced social bullying. About $27 \%$ experienced physical bullying and $13.3 \%$ experienced internet bullying.

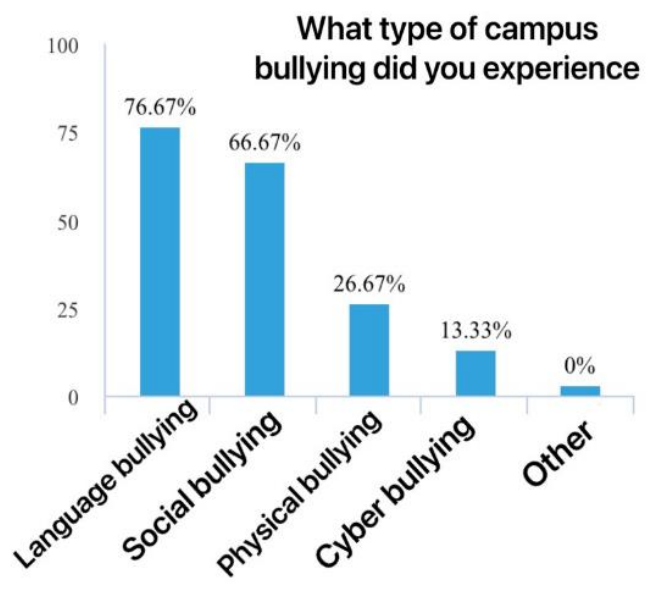

Figure 1 Types of campus bullying 


\subsection{Desirability (Test hypothesis1)}

Hypothesis 1 is accepted that people who have experiences with school bullying want to have a platform to express their emotion. The attitudes toward our platform are collected. The right part of Figure 2 shows that when people were asked if they wanted a platform to chat with people who had the same experience, $66.7 \%$ of them wanted it. In the left part of Figure 2, the statistic results are about whether people need a platform to provide them with our professional psychological consultation. In this set of data, people who "wanted" and "wanted very much" account for $80 \%$ of the data. This data shows that currently, there is a potential market for an app designed to deal with campus bullying. The emergence of APP will undoubtedly attract a lot of attention, and there will be many businesses willing to sponsor us, considering possible large consumer base.

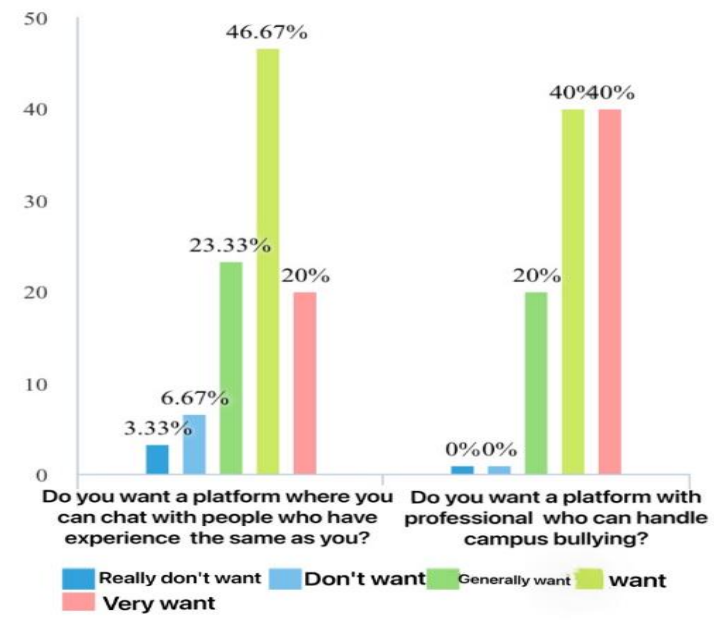

Figure 2 Market potential

\subsection{Viability}

Hypothesis 2 is rejected as schools are not willing to fund, according to 4 teachers who came from public school, international schools. When it comes to the remedy of mental harm to victims, most teachers expressed the schools were reluctant to pay for the APP platform, on which the trauma of school violence victims can be healed with the help of professional psychologists. There are a few points of why schools wouldn't pay:

1) The school has not aroused adequate attention to school violence as students who are bullied usually do not choose to report this to teachers.

2) Schools do not have the decision power to fund app development. Only the higher-level authorities, like the Education Bureau and the government, can make relevant instructions. The whole communication cost is huge and takes a lot of time to reach an agreement.

According to teachers, campus violence still occurred frequently in schools; however, the teachers had hardly encountered the report of school violence from students in their teaching career. If school violence was reported, the school's solution was generally divided into two categories. When the violence issue was mild which is mild physical injury, the class teacher would educate the bullies in verbal form. When the violence degree was uplifted to a more serious level which is serious injuries may require medical treatment, the bullies would have to drop out of school or the public security agency would intervene.

Hypothesis 3 - victims of school bullying are willing to pay 30 yuan per month for the services, is rejected. We investigated the payment intentions of the respondents among the bullied. In Figure 3, we are surprised to find that $23.3 \%$ of the people who had experienced campus bullying were unwilling to pay. This shows that they have not realized the seriousness of campus bullying, and 70\% of the respondents were only willing to pay a fee of less than $30 \mathrm{RMB}$, which shows that people are only willing to use very little money to solve their psychological problems. We see that it is not enough to rely solely on platform operations to make money. We need other payment methods such as advertising bidding, offline event promotion, etc. to maintain platform operations.

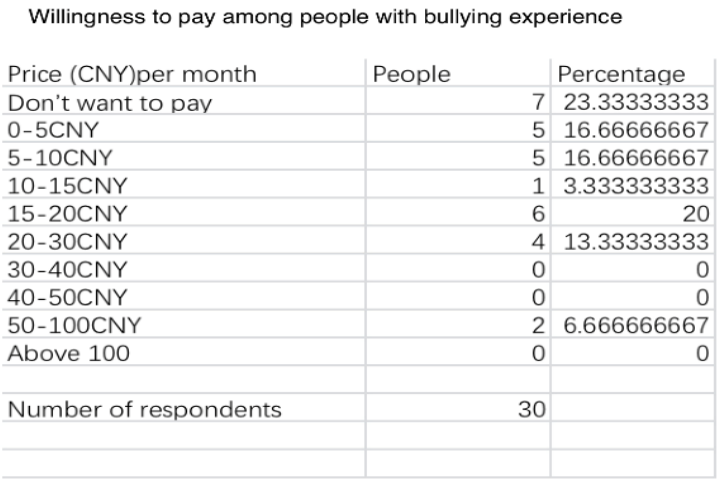

Figure 3 Willingness to pay among victims

\subsection{Feasibility (Hypothesis 4)}

To get insights on the psychological counselling services, we interviewed one psychologist about the price of services. We learned that psychological counselling and psychotherapy are currently priced in the market, and the government no longer sets prices uniformly. Public hospitals charge cheaper than private hospitals. In private hospitals, for example, Guangzhou Huia Hospital's psychological treatment is $200 \mathrm{RMB} /$ half-hour and 600$800 \mathrm{RMB}$ for psychological treatment. In public hospitals, for instance, Guangzhou First People's Hospital offers 50 $\mathrm{RMB} /$ half-hour for psychological consultation and 400 $\mathrm{RMB} /$ hour for psychological treatment. These data might help us to further estimate the pricing of our app. Then we learned that these hospitals are difficult for patients to register, because there are not many outpatient numbers for psychological consultation and treatment. 
We further inquired about other platforms' commission and found that telephone consultations with doctors are usually $100 \mathrm{RMB}$ for 15 minutes, and the platform may take about $20 \%$ of the commission.

Although we gain some information for the psychological counselling market in China from this psychologist, due to limited access to other psychologists and the secrecy about psychological counselling, hypothesis 4 is underexplored in this research and further investigation is needed.

\subsection{Platform design}

\begin{tabular}{l|l|r|}
$\begin{array}{l}\text { What feature do you think you are most likely to pay for? } \\
\text { Option }\end{array}$ & Data & Percentage \\
\hline Voice call with specified friends & 13 & 12.38095238 \\
\hline Message to designated friends & 11 & 10.47619048 \\
\hline Consult a professional psychologist & 91 & 86.66666667 \\
\hline Personalized setting (clothes,background) & 17 & 16.19047619 \\
\hline Anonymous & 10 & 9.523809524 \\
\hline Modify positioning & 4 & 3.80952381 \\
\hline Increase post exposure & 19 & 18.0952381 \\
\hline Other suggestion & 2 & 1.904761905 \\
\hline Number of valid entries in this question & 105 & \\
\hline
\end{tabular}

Figure 4 Features to pay

As shown in Figure 4, we asked the respondents' preferred paid features. Professional psychologist consultation obtained the most votes, 91 out of 105 votes, which indicated that consultation might be the main focus of the platform.

We collected data from people who have recovered from their bully experience whether they are willing to help others on the platform and their motivations to help. According to Figure 5,69.3\% of the interviewees were willing to help. Furthermore, $69.2 \%$ thought that they didn't need any rewards to help these people (Figure 6). These are very optimistic data for us as they let us know that the majority of people on our platform are willing to help those who are in deep pain for free.

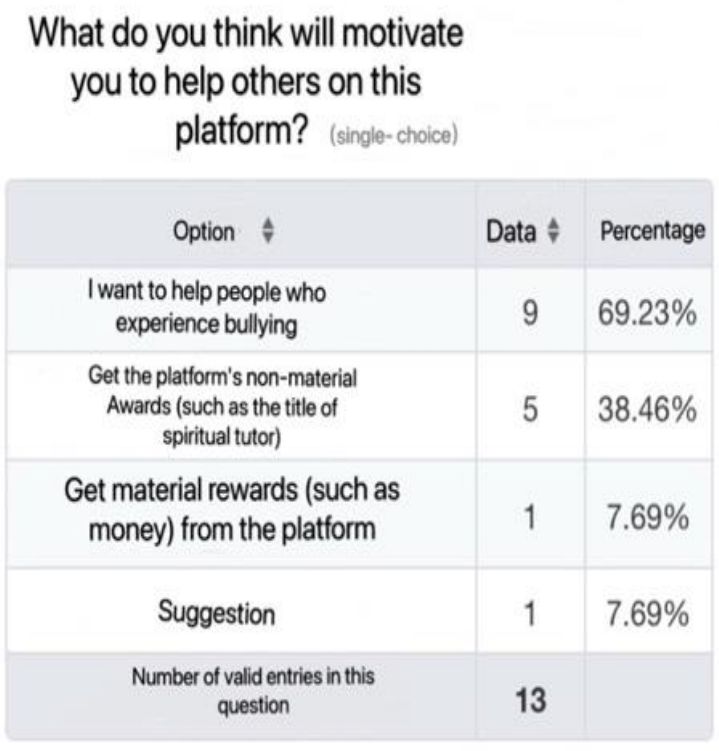

Figure 5 Recovered victims' willingness to help

\section{Do you have emotions that you want to express anonymously}

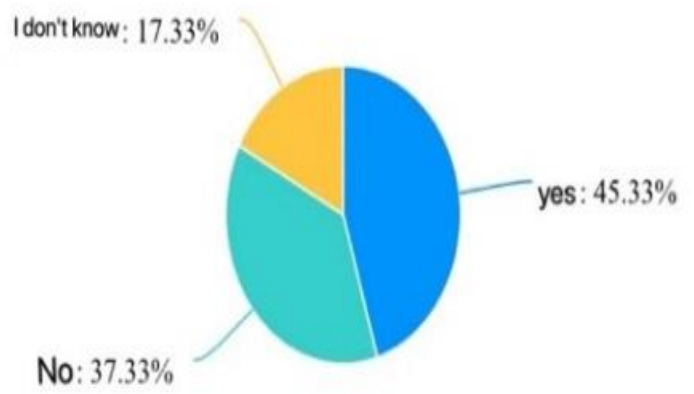

Figure 6 Motivations of helping

Information privacy is an important consideration. In view of the long-term development of this platform, we are bound to face transformation and broaden the customer base, so we need to discover more potential customers. In addition to the current campus bullying, we need to explore more services that are based on psychological counselling. Therefore, we asked whether those who had not been bullied also wanted to express their emotions anonymously. As shown in Figure 7, 45.3\% of them thought they needed it. For such a platform, this data allows us to discover our potential customers so that when we refine this platform, we can group nearly half of them into our customer groups. 


\begin{tabular}{|c|c|c|}
\hline \multirow[b]{2}{*}{ Option $*$} & \multicolumn{2}{|c|}{ (Single-choice) } \\
\hline & Data $*$ & Percentage \\
\hline Really don't want & 1 & $7.69 \%$ \\
\hline Don't want & 0 & $0 \%$ \\
\hline Doesn't matter & 3 & $23.08 \%$ \\
\hline Want & 6 & $46.15 \%$ \\
\hline Really want & 3 & $23.08 \%$ \\
\hline $\begin{array}{l}\text { Number of valid entries in this } \\
\text { question }\end{array}$ & 13 & \\
\hline
\end{tabular}

Figure 7 Privacy concern

Moreover, in Figure 8, the interviewees' prior choice was private text chat $-43.9 \%$. Through the result of ranking, we understand that the respondents are very privacy-conscious. Both user information and their chat content should be protected well with a well-developed scheme of privacy protection on our platform.

\begin{tabular}{|c|c|c|}
\hline $\begin{array}{c}\text { prior choice } \\
\text { choice }\end{array}$ & Subtotal & ratio \\
\hline $\begin{array}{c}\text { Regional text chat } \\
\text { Private text chat }\end{array}$ & 40 & $37.38 \%$ \\
\hline $\begin{array}{c}\text { Private voice chat } \\
\text { 3D virtual chat (withyour own } \\
\text { character) }\end{array}$ & 6 & $53.93 \%$ \\
\hline Other & 13 & $12.15 \%$ \\
\hline Number of valid entries & 107 & $0.93 \%$ \\
\hline
\end{tabular}

Figure 8 Interactive modes

\section{CONCLUSION}

The research found supportive evidence for the participants' acceptance and wants of the designed platform. However, the study found that schools would not fund the development of the application and participants had low expected monthly cost for the application.

The desirability of a friend-making online platform for school bullying victims is relatively high, which indicates that people who experienced peer victimisation wishes and need a platform to share experience and gain support from others. Moreover, professional psychological counselling services are preferable by most victims which indicates victims' needs in professional guidance and interventions, which are rarely found in the Chinese contexts. Therefore, there is a market opportunity for the application as there is a gap between people's needs for interventions and limited effective intervention programmes in Chinese schools derived from the conversations with teachers.

Two main reasons were identified for the schools' unwillingness to fund, schools' unconsciousness of school bullying situations, and legitimacy of bill-paying. Our suggestions to the future investigation are to test the desirability of the government which have greater power or more legitimate to fund the application as schools follow the government policies to deal with school bullying.

Expected income from users was low with most participants were pay less than 30 yuan per month. Further investigation is needed for the reasons for the low expected cost of consumers when compared to the relatively high fees for psychological counselling services. One reason might be our participants have limited knowledge of the service price in real life. Another possibility might be victims of school bullying are mostly school-aged or university students without relatively low income, which means services are unaffordable for victims. However, nearly $70 \%$ of recovered victims demonstrated their willingness to voluntarily participate in the mentoring process for school bullying victims. Therefore, the expected expenditure of the mentoring services might be lowered down. This study explored interviewees' preferred platform design and found that they are more likely to pay for professional psychological consultation. Recovered victims expressed high willingness to help without material or non-material awards. In terms of privacy, about $45 \%$ of interviewees valued anonymity and preferred to use private text chat.

In terms of the limitation of the research, the second feasibility and viability risks of this social venture are underexplored and future investigations are needed. The reason for the under-exploration is the limited access to relevant samples. Due to the position of the psychologist, limited information was gathered about the willingness of the wider community of therapists and personal income through online counselling services. Moreover, due to limited capacity, many risks related to the feasibility, viability and desirability are not tested in this research, such as the cooperation willingness of the government. Additional research involves conversations and surveys with supply and demand sides will be helpful.

\section{REFERENCES}

[1] Rigby K. 2000. Effects of peer victimisation in schools and perceived social support on adolescence 
wellbeing. J Adolesc, 23:57-68.

[2] Hawker DS, Boulton MJ., 2020, Twenty years' research on peer victimization and psychosocial maladjustment: A meta-analytic review of crosssectional studies. Journal of Child Psychology and Psychiatry. 41(4):441-455.

[3] Chan, H.C.; Wong, D.S.W. Traditional school bullying and cyberbullying in Chinese societies: Prevalence and a review of the whole-school intervention approach. Aggress. Violent Behav. 2015, 23:98-108

[4] Chang F-C, Lee C-M, Chiu C-H, Hsi W-Y, Huang TF, Pan Y-C. Relationships among cyberbullying, school bullying, and mental health in Taiwanese adolescents. J Sch Health. 2013; 83: 454-462.

[5] Han, Z., Zhang, G. and Zhang, H., 2017. School bullying in urban China: Prevalence and correlation with school climate. International journal of environmental research and public health, 14(10):1116.

[6] Zhang, W.,2016, "Interventions against bullying in mainland China," in Smith, P. K., Kwak, K., and Toda, Y. (eds) School Bullying in Different Cultures: Eastern and Western Perspectives. Cambridge: Cambridge University Press, 365-375.

[7] Ministry of Education of the People's Republic of China, 2018, Guidebook for Combating Bullying and Violence among Primary and secondary school students.

[8] Xu, F., Xu, J. and Huang, D., 2016. On the Legalized Governance of School Bullying. Educational Research and Experiment, (6):50-53

[9] Sina News, 2020. Representative of Li Yalan: It is suggested to legislate separately to refine punishment standards for school bullies. [Online]. Available at: https://news.sina.com.cn/c/2020-0521/doc-iirczymk2771618.shtml.

[10] Chinanews, 2020. The Underage Protection Act defines "student bullying" for the first time, experts say. [Online]. Available at: http://www.chinanews.com/gn/2020/1029/9325074.shtml.

[11] Yeung Thompson, R.S. and Leadbeater, B.J., 2013. Peer victimization and internalizing symptoms from adolescence into young adulthood: Building strength through emotional support. Journal of research on adolescence, 23(2):290-303. 